\title{
Eficácia da memantina na doença de Alzheimer em seus estágios moderado a grave
}

\author{
Efficacy of memantine in moderate to severe Alzheimer disease
}

Rosana Soares Araújo e Milena Pereira Pondé

\begin{abstract}
Resumo
Introdução: O objetivo desta revisão de literatura é avaliar a eficácia, a segurança e a tolerabilidade da memantina, um antagonista não-competitivo do receptor $\mathrm{N}$-metil-D-aspartato (NMDA), no tratamento da doença de Alzheimer em seus estágios moderado a grave. Métodos: Realizou-se uma busca no banco de dados MEDLINE com as palavras-chave memantine $e$ Alzheimer's disease, sendo inseridos apenas ensaios clínicos randomizados, duplo-cegos e controlados com placebo. Resultados: Foram incluídos quatro estudos que preenchiam os critérios de inclusão supracitados, realizados com pacientes portadores de doença de Alzheimer com grau moderado a grave. Todos os estudos indicaram um efeito benéfico da memantina com relação ao placebo para os seguintes parâmetros: melhora da capacidade funcional e maior participação nas atividades diárias. Dois estudos evidenciaram melhora cognitiva. A duração média dos estudos foi de 28 semanas e as doses mais eficazes variaram de 10 a $20 \mathrm{mg} / \mathrm{dia}$. Os efeitos adversos, em todos os estudos, foram maiores no grupo placebo. Discussão: Apesar de ser uma droga nova e ainda de custo elevado, a memantina parece reduzir os custos totais e o tempo gasto do cuidador, além de produzir melhora global do paciente, gerando melhor qualidade de vida tanto para o paciente quanto para o cuidador. Estudos ainda não publicados, contudo, sugerem que o impacto dessa droga nos estágios mais avançados da demência de Alzheimer seja marginal.
\end{abstract}

Palavras-chave: memantina, Alzheimer, tratamento.

\begin{abstract}
Introduction: The aim of this systematic review is to evaluate efficacy, safety and tolerability of memantine, a non-competitive agonist of $\mathrm{N}$-methyl-D-aspartate (NMDA) receptor, on the treatment of moderate to severe Alzheimer disease. Methods: A search in MEDLINE database was made using key words memantine and Alzheimer's disease. Only randomized, double-blinded and placebo controlled clinical trials were included. Results: Four studies were found that fulfilled inclusion criteria; all patients had moderate or severe Alzheimer disease. All studies suggested that memantine is superior to placebo for the following parameters: improvement in functional abilities and more participation in daily activities. Two studies suggestted cognitive improvement. Mean duration of studies was 28 weeks, and doses of memantine ranged between 10 to $20 \mathrm{mg} / \mathrm{bid}$. Side effects, in all studies, were worst for placebo group. Discussion: In spite of its costs, memantine seems to reduce total costs and time spent by caregiver, and improves patient and caregiver quality of life.
\end{abstract}

Key words: memantine, Alzheimer, treatment. 


\section{Introdução}

A doença de Alzheimer (DA) é um transtorno neurodegenerativo progressivo, manifestado por deterioração da memória, da cognição e das atividades da vida diária, associado a uma variedade de sintomas psiquiátricos e a distúrbios comportamentais. Essa doença afeta aproximadamente 15 milhões de pessoas no mundo (Khachaturian, 1997), porém o número de pessoas acometidas aumenta substancialmente com a idade da população (Henderson e Jorm, 2002). Tratamentos farmacológicos são utilizados freqüentemente para as fases leve a moderada da doença de Alzheimer, não havendo opções para os estágios mais avançados dessa enfermidade. 0 glutamato é o principal neurotransmissor excitatório do cérebro (Fonnum, 1984; Orrego e Villanueva, 1993).

Uma alta estimulação glutaminérgica pode resultar em prejuízo neuronal, fenômeno que tem sido denominado excitotoxicidade. A memantina é a primeira de uma nova classe de drogas para os estágios moderado a grave da DA. É uma droga antagonista, não-competitiva, voltagem-dependente do receptor N-metil-D-aspartato (NMDA), que bloqueia os efeitos patológicos dos níveis elevados de glutamato (Danysz et al., 2000). O objetivo desta revisão de literatura é avaliar a eficácia, a segurança e a tolerabilidade do uso da memantina em pacientes portadores da doença de Alzheimer nos estágios moderado a grave.

\section{Métodos}

As bases de dados utilizadas para esta revisão de literatura foram MEDLINE, Literatura Latino-Americana e do Caribe em Ciências da Saúde (LILACS), e Education Resources Information Center (ERIC) para consultar artigos não-publicados e trabalhos apresentados em conferências; o University Microfilms International Dissertation Service para consultar dissertações e teses não-publicadas; o Sistema de informação da Biblioteca da Organização Mundial da Saúde (WHOLIS), bem como o National Technical Information Service (NTIS), que resume estudos subvencionados por mais de 600 agências federais dos EUA.

As palavras de busca utilizadas foram memantine e Alzheimer's disease, sendo incluídos apenas estudos feitos em humanos, com desenho duplo-cego, randomizado e placebo- controlado. Os artigos deveriam ter pontuação superior a 4, de acordo com os critérios de Jadad (Jadad et al., 1996). Essa pontuação compreende:

- um ponto para estudo randomizado;

- um ponto se a randomização foi feita adequadamente;

- um ponto se o estudo é duplo-cego;

- um ponto se o método duplo-cego foi descrito e é apropriado;

- um ponto se são descritos os dropouts.

Foram excluídos os relatos de caso, os estudos abertos, as revisões de literatura e as metanálises.

\section{Resultados}

Com as palavras de busca usadas foram encontrados no MEDLINE 55 artigos entre os anos de 1999 e 2006 . Feita a restrição para ensaios clínicos duplo-cegos, randomizados e placebo-controlados, restaram seis artigos. Dois desses foram excluídos, pois compreendiam o tratamento da demência vascular, a DA associada a co-morbidades neurológicas e a associação da memantina com outras drogas. Foram inseridos quatro artigos, com desenho duplo-cego, randomizado e placebo-controlado e com pontuação superior a 4, de acordo com os critérios de Jadad (Jadad et al., 1996). Os pacientes avaliados foram classificados como portadores da doença de Alzheimer de moderada a grave, de acordo com as suas pontuações em diversas escalas. A Tabela 1 mostra uma síntese dos principais resultados dos estudos selecionados.

Reisberg et al. (2003) (Jadad = 5) realizaram um estudo randomizado, duplo-cego e controlado com placebo, num período de 28 semanas, em que foram envolvidos 252 pacientes portadores da DA de grau moderado a grave, diagnosticados de acordo com o Diagnostic and Statistical Manual of Mental Disorders IV (DMS-IV). Os pacientes tiveram o Mini-Mental State Examination (MMSE) com pontuação entre 3 e 14 e uma média de 7 a 9; Global Deterioration Scale (GDS) no estágio 5 ou 6; e Functional Assessment Staging (FAST) em estágio de 6a.

Pacientes com demência vascular, desordens neurológicas, co-morbidades clinicamente significantes, depressão maior e/ou exames laboratoriais anormais foram excluídos, além daqueles que recebiam medicações concomitantes, como agentes anticonvulsivantes, antiparkinsonianos, hipnóticos, ansiolíticos, neurolépticos ou colinomiméticos. Trata-se de um estudo com randomização realizada por computador, através

Tabela 1. Sumários dos estudos selecionados

\begin{tabular}{|c|c|c|c|c|c|c|}
\hline Autor/ano & $\begin{array}{c}\mathrm{N}^{0} \text { de } \\
\text { pacientes }\end{array}$ & Tempo do estudo & Dose & $\begin{array}{l}\text { Escala de } \\
\text { avaliação }\end{array}$ & $\begin{array}{c}\text { Resultado } \\
\text { Memantina vs. placebo }\end{array}$ & $p$ \\
\hline Winblad et al., 1999 & 79 & 12 semanas & $10 \mathrm{mg}$ & CGI-C* & $\begin{array}{c}\text { Melhora de } 30 \text { pacientes }(73 \%) \text { no grupo me- } \\
\text { mantina contra melhora de } 16 \text { pacientes }(42 \%) \\
\text { no grupo placebo }\end{array}$ & $<0,0005$ \\
\hline Wimo et al., 2003 & 252 & 28 semanas & $20 \mathrm{mg}$ & CIBIC-Plus ${ }^{* *}$ & $\begin{array}{l}\text { Média de diferença de } 0,36 \text { favorecendo } \\
\text { o grupo da memantina }\end{array}$ & $<0,025$ \\
\hline Reisberg et al., 2003 & 252 & 28 semanas & $20 \mathrm{mg}$ & CIBIC-Plus & $\begin{array}{c}\text { No grupo placebo houve aumento } \\
\text { da escala para } 4,7 \text { e no grupo } \\
\text { memantina, para } 4,4\end{array}$ & $<0,003$ \\
\hline Rive et al., 2004 & 252 & 28 semanas & $20 \mathrm{mg}$ & CIBIC-Plus & $\begin{array}{l}\text { Maior autonomia no grupo memantina, tanto em } \\
\text { pacientes autônomos como em dependentes }\end{array}$ & $\begin{array}{l}<0,006 \\
<0,035\end{array}$ \\
\hline
\end{tabular}

${ }^{*} \mathrm{CGI}-\mathrm{C}$ (Clinical Global Impressiono f Change); **CIBIC-Plus (Clinician's Interview-Based Impression of Change Plus Caregiver Input). 
do Ran Code (versão 3.1), em que 126 pacientes receberam memantina numa dose de $20 \mathrm{mg}$ ao dia e 126 fizeram uso de placebo por um período de 28 semanas.

Para avaliar a evolução dos pacientes foram utilizados os seguintes instrumentos: Clinician's Interview-Based Impression of Change Plus Caregiver Input (CIBIC-Plus), Alzheimer's Disease Cooperative Study Activities of Daily Living Inventory (ADCS-ADLsev), Severe Impairment Battery (SIB), FAST, MMSE e GDS.

O CIBIC-Plus avalia sistematicamente o domínio da cognição, função e comportamento do paciente. A elevação da pontuação indica piora do quadro. Os pacientes que receberam placebo tiveram piora progressiva ao final de 28 semanas, comparados com os que receberam memantina. Todos os pacientes tinham escore de 4 no CIBIC-Plus, contudo nos que receberam placebo os escores se elevaram para aproximadamente 4,7 e nos que receberam memantina, para 4,4. A diferença foi estatisticamente significativa $(p<0,03)$ ao final das 28 semanas; entretanto, com a continuação da observação, a diferença não foi estatisticamente significante $(p<0,06)$.

O ADCS-ADLsev, modificado para o estado grave, determina a habilidade do paciente para a sua performance nas atividades da vida diária. 0 total de 54 pontos significa ótima performance, e pontos baixos indicam piora. Essa escala foi pontuada, no início do estudo, em 27,4 no grupo placebo e 26,8 no grupo memantina. Foi observado que, ao final das 28 semanas, houve menor deterioração do grupo que recebeu memantina de aproximadamente -3 , em comparação ao grupo placebo, com aproximadamente -6 , sendo essa diferença estatisticamente significante $(p<0,003)$. Com a contínua observação essa diferença se reduziu, porém permaneceu estatisticamente significativa $(p<0,02)$.

A avaliação feita pelo SIB observa a performance cognitiva da DA, incluindo interação social, memória, linguagem, habilidade visual, atenção, praxia e construção, com pontuação que varia de 0 (considerável deterioração) até 100. Essa escala favoreceu o grupo que recebeu memantina, sendo que ao final das 28 semanas houve decréscimo na escala do grupo placebo de aproximadamente dez pontos, comparado com decréscimo de aproximadamente cinco pontos no grupo memantina $(p<0,002)$. A diferença permaneceu significante com a contínua observação $(p<0,001)$. Pacientes tratados com memantina mostraram-se significativamente menos deteriorados, quando comparados com o grupo placebo $(p<0,007)$, ao final de 28 semanas. Os resultados de todas as escalas se mantiveram significantes no end point, exceto o CIBIC-Plus.

Por fim, nas análises da população estudada ao final das 28 semanas, não foram observadas diferenças significativas entre os grupos placebo e memantina nas escalas MMSE, GDS ou Neuropsychiatric Inventory (NPI). Foram registradas 71 exclusões, sendo 42 pacientes no grupo placebo e 29 no grupo da memantina. Agitação foi a razão mais prevalente da descontinuidade do estudo, apresentando-se em $7 \%$ dos que receberam placebo e $5 \%$ dos que receberam memantina. Efeitos adversos sérios foram reportados em 23 pacientes (18\%) que receberam placebo e 16 que receberam memantina (13\%). Sete mortes ocorreram durante o estudo, apenas duas no grupo memantina. Os efeitos adversos mais sérios incluídos em todas as mortes não foram considerados relacionados com o uso da medicação. A ocorrência de pelo menos um evento adverso relacionado com o tratamento foi mais freqüente no grupo placebo (87\%), quando comparado com o grupo memantina (84\%). Os efeitos colaterais mais freqüentes reportados foram agitação, incontinência urinária, insônia e diarréia. Os dois últimos efeitos foram mais freqüentes no grupo da memantina. Não houve diferença significativa nos valores laboratoriais, nos eletrocardiogramas (ECG) e nos sinais vitais durante o estudo entre os dois grupos.

Rive et al. (2004) (JADAD = 4) realizaram um estudo duplo-cego, randomizado e placebo-controlado, com uma amostra inicial de 252 pacientes, obedecendo aos critérios para definir o estágio da doença de Alzheimer, de acordo com a escala do MMSE (MMSE $\geq 10$, moderadamente grave, e $<10$, grave). 0 estudo realizou uma triagem clínica para avaliar a eficácia e a tolerabilidade de $20 \mathrm{mg}$ de memantina por dia, comparada com placebo, por um período de 28 semanas. A seleção dos pacientes foi realizada pelo ADCS-ADLsev, sendo todos classificados de acordo com as capacidades básicas (basic activities of daily living [BADL]) e instrumental (instrumental activities of daily living [IADL]). As atividades básicas são simples, como comer, vestir-se sozinho, transferir-se e utilizar o banheiro de forma independente, enquanto as atividades instrumentais são mais complexas, como utilizar o telefone, preparar comida e/ou lavar objetos. A função cognitiva foi mensurada pelo MMSE e pelo SIB.

Para os sintomas comportamentais foi utilizado o NIP e para o grau da severidade, o FAST e o GDS. O CIBIC-Plus foi usado para verificar o resultado primário da eficácia da triagem clínica. 0 questionário The Resource Utilization in Dementia (RUD) (Wimo et al., 1998) foi utilizado para mensurar o tempo gasto (por mês) pelos cuidadores para assistir aos pacientes. Os 252 pacientes incluídos na triagem foram divididos em dois grupos. A classificação dos dois grupos, computada por algoritmos, formulou os critérios de autonomia para cada paciente. $O$ número de grupos foi determinado pelo pseudo $F$ estatístico. 0 grupo 1 foi composto por 146 pacientes dependentes, pois possuíam as menores pontuações, de acordo com as classificações supracitadas. 0 grupo 2 foi composto por 106 pacientes com certa autonomia. 0 critério de autonomia foi definido pela seguinte equação: IADL + 1,6 x BADL. Com resultado $>32,4$, o paciente é considerado autônomo e, caso contrário, é dependente. Não houve diferença significativa entre autônomos e dependentes com relação a sexo ou idade. A duração da doença foi maior para os pacientes dependentes (média de 5,8 anos $\pm 3,4$ ) do que para os autônomos (média de 5,1 anos $\pm 3,9)(p=0,033)$. 0 início da doença antes dos 65 anos não mostrou diferença significante entre pacientes autônomos e dependentes.

Uma diferença estatisticamente significante entre pacientes autônomos e dependentes foi observada nas escalas MMSE, SIB, FAST, GDS e NPI, todas em favor dos pacientes autônomos, indicando que esses têm menos distúrbios cognitivos, funcionais e comportamentais $(p<0,001)$. 0 tempo dos cuidadores e os custos são maiores nos pacientes dependentes $(p<0,001)$. Além disso, a proporção da institucionalização é maior em pacientes dependentes (10,5\%) quando comparados com os autônomos $(5,7 \%)$; contudo, a diferença 
não é estatisticamente significante. Os pacientes autônomos tratados com memantina tiveram, em seis meses, uma autonomia maior, quando comparados com os que receberam placebo (razão de risco $[\mathrm{OR}]=3,03$; intervalo de confiança [IC]: 95\%, 1,38-6,66). Nos pacientes com doença moderamente grave e grave, os dependentes, essa diferença foi menor, mas se manteve estatisticamente significante no mesmo período de tempo (OR = 2,22; IC: 95\%, 1,06-4,65).

Resultados similares foram identificados com a utilização do Treated Per Protocol (TPP), com 78 pacientes no grupo placebo e 93 no grupo memantina, e com o método Last Observation Carried Forward (LOCF), em que 247 pacientes obtiveram uma última análise pelo ADCS-ADLsev. Em ambos os casos a memantina foi benéfica na melhora da autonomia dos pacientes ao final das 28 semanas ( $p<0,024$ e $p<0,023)$, reduzindo assim os custos e diminuindo a responsabilidade do cuidador. Os efeitos colaterais mais freqüentes foram agitação e insônia, com predomínio no grupo placebo, porém não houve diferença estatisticamente significante entre os grupos placebo e memantina.

Winblad e Poritis (1999) (Jadad $=4$ ) realizaram um estudo placebo-controlado, duplo-cego e randomizado, com uma amostra inicial de 167 pacientes. Foram selecionados pacientes de ambos os sexos, idades entre 60 e 80 anos, com demência definida pelo Diagnostic and Statistical Manual of Mental Diseases III (DSM-III-R, 1987). Outros critérios de inclusão foram os estágios 5-7 do GDS e MMSE < 10 pontos, com duração da demência superior a 12 meses. Os pacientes excluídos do estudo possuíam algum tipo de enfermidade não controlada, como doença terminal, insuficiência cardíaca progressiva, hipertensão grave, infarto do miocárdio nos últimos três meses, arritmias cardiacas graves, diabetes mellitus (DM) instável, doença hepática crônica, deterioração renal grave (nível sérico de creatinina $>2 \mathrm{mg} \%$ ), deterioração da função tireoidiana, doença psiquiátrica grave (esquizofrenia e depressão maior), epilepsia e/ou doença de Parkinson, bem como os que estavam em uso de outras drogas como inibidores da monoamina oxidase (IMAOs), neurolépticos, antidepressivos tricíclicos, hipnóticos, nootrópicos ou agentes estimuladores da circulação cerebral.

A avaliação dos resultados foi feita em dois tempos Os critérios de primeira eficácia eram compostos pelo Clinical Global Impression of Change ( $\mathrm{CGI}-\mathrm{C}$ ), que avalia a mudança e a performance do paciente com uma impressão clínica global, realizada pela experiência do clínico, independente da avaliação feita pelo cuidador, e pelo Behavioral Disturbances of Geriatric Patients (BGP), que observa capacidade funcional e distúrbios comportamentais dos pacientes geriátricos acompanhados pela equipe de enfermagem. O BGP é composto por quatro subescalas com 35 itens, sendo que esse estudo utilizou a subescala de dependência de cuidados. As variáveis da segunda eficácia foram classificadas de acordo com o $D$-Scale, que tem dezesseis variáveis funcionais como critérios mais importantes para caracterizar a independência, classificados desde uma função normal até um cuidado dependente total.

Os resultados apresentados nesse estudo foram realizados com pacientes portadores de DA, demência vas- cular e mista. Uma amostra de 79 pacientes representava os portadores de DA que foram randomizados em grupos, sendo 41 pacientes em uso de $10 \mathrm{mg}$ de memantina ao dia e $38 \mathrm{em}$ uso de placebo. Na avaliação do CGI-C, após 12 semanas, 30 pacientes $(73 \%)$ em uso de memantina responderam com melhora e apenas 11 pacientes (27\%) não responderam ao uso da droga. 0 grupo placebo obteve resposta em 16 pacientes $(42 \%)$, evidenciando fortemente o envolvimento de fatores psicológicos, e 22 pacientes (58\%) não obtiveram resposta. A diferença entre os dois grupos foi estatisticamente significante $(p<0,0005)$. Na avaliação do BGP, os pacientes que receberam memantina tiveram diminuição da dependência $(-3,5 \pm 12,5)$ mais acentuada quando comparados com os pacientes do grupo placebo $(-1,6 \pm 9,2)(p=0,016)$.

0 estudo evidenciou que, em relação à segurança e à tolerabilidade, 18 pacientes $(22 \%)$ do grupo memantina e 18 pacientes $(21 \%)$ do grupo placebo tiveram efeitos adversos. Quatro pacientes (5\%) do grupo memantina e cinco pacientes $(6 \%)$ do grupo placebo tiveram efeitos adversos sérios, que consistiram em piora da insuficiência cardíaca, apnéia, distúrbio circulatório cerebral, parada cardíaca, coma, hospitalização com dor abdominal, febre e vômitos, entre outros. Em todos os casos dos efeitos adversos sérios não foi observada relação com a medicação. A tolerância da medicação foi evidenciada pelos parâmetros laboratoriais, mostrando que $71 \%$ dos pacientes do grupo memantina e $69 \%$ do placebo toleraram muito bem o tratamento; $28 \%$ do grupo memantina e $31 \%$ do placebo mostraram boa tolerabilidade; e apenas um paciente do grupo memantina apresentou moderada tolerabilidade.

A freqüência de valores laboratoriais anormais foi distribuída, de forma equivalente, em ambos os grupos. Dessa forma, esse estudo evidenciou que o tratamento com a memantina, na dose de $10 \mathrm{mg} / \mathrm{dia}$, por um período superior a 12 semanas, melhora a capacidade funcional, a necessidade dos cuidados de dependência e os sintomas comportamentais de demência grave, além de a droga ser bem tolerada e segura.

Wimo et al. (2003) (Jadad $=4$ ) realizaram um estudo multicêntrico, duplo-cego, randomizado e placebo-controlado, por um período de 28 semanas, com triagem clínica feita nos EUA. Os pacientes selecionados tinham idade mínima de 50 anos com provável DA, de acordo com o DMS-IV e o critério do National Institute for Communicative Disorders and Stroke - Alzheimer's Disease and Related Disorders Association (NINCDS/ADRDA) (McKhann et al.,1984). Os critérios elegíveis incluíram as pontuações de 3-14 do MMSE, os estágios 5 ou 6 do GDS e o estágio $6 a$ do FAST. As análises dos efeitos da medicação na função e na cognição do paciente foram realizadas pelas escalas ADCS-ADLsev, FAST, SIB, e a relevância clínica foi confirmada pelo CIBIC-Plus.

O estudo também evidenciou a utilização de recursos, que foram mensurados pelos gastos com enfermagem em casa, no hospital ou em emergências, bem como com as horas gastas pelo cuidador informal, usando o questionário RUD durante os eventos ocorridos em um mês, e um seguimento dos eventos ocorridos em três meses. 0 questionário foi completado nas semanas 12 e 28, ou quando os pacientes terminavam o estudo prematuramente. Os 
eventos específicos do questionário incluíam tempo empregado pelo cuidador para o paciente, mudança de trabalho do cuidador, utilização de recursos para cuidados com a saúde tanto por parte do paciente quanto por parte do cuidador, e posição residencial do paciente, caracterizada no fato de morar na comunidade ou em instituição. Todas as variáveis foram calculadas por um período de seis meses; entretanto o tempo do cuidador foi calculado em sua totalidade por apenas dois meses.

A análise dos custos foi calculada com base nas despesas diretas com medicamento, gastos não-medicamentosos e tempo do cuidador. Os 252 pacientes foram randomizados, sendo que 126 receberam $10 \mathrm{mg}$ de memantina duas vezes ao dia, totalizando $20 \mathrm{mg} /$ dia e 126 receberam placebo. Apenas 181 (97 no grupo memantina e 84 no grupo placebo) completaram o período de 28 semanas de tratamento. A não-continuidade foi mais alta no grupo placebo (42 pacientes, $33 \%$ ) do que no memantina (29 pacientes, $23 \%$ ). A razão mais freqüente para esse evento foram alguns efeitos adversos, como a agitação, que também foi maior no grupo placebo (7\%) do que no memantina (5\%).

Ao final das 28 semanas a superioridade da memantina em relação ao placebo foi estatisticamente significante para os domínios global, funcional e cognitivo. O CIBIC-Plus encontrou uma média de diferença de 0,36 pontos nos 181 pacientes analisados (97 no grupo memantina e 84 no placebo), favorecendo os pacientes que utilizavam memantina e revelando menor aumento na escala, quando em comparação com o grupo placebo $(p<0,025)$. Na avaliação funcional realizada pelo ADCS-ADLsev, a média de diferença entre os grupos, favorecendo a memantina, foi de 3,37 pontos $(p=0,003)$ com a mesma amostra supracitada. 0 total de pontos mensurado pelo SIB evidenciou consistente efeito da memantina no desempenho cognitivo, com uma diferença de pontos de 5,7 ( $p=0,002)$ em uma amostra de 179 pacientes.

Nas escalas MMSE e NPI não houve diferença significativa entre os grupos ao final das 28 semanas. As diferenças de importância clínica em relação aos efeitos adversos não foram consideradas significativas entre os grupos. 0 tempo dos cuidadores dos pacientes que receberam memantina foi significativamente menor do que os cuidadores do grupo placebo, com uma diferença de 51,52 horas por mês $(p=0,02)$. Análises adicionais evidenciadas pelo LOCF e pela intenção de tratar (ITT) populacional avaliaram o tempo gasto pelo cuidador e confirmaram resultado similar ao do TPP, sendo observada superioridade estatisticamente significativa da memantina em relação ao placebo, com uma diferença de 45,82 horas por mês $(p=0,01)$. 0 total de custos dos cuidadores e fatores sociais foram estatisticamente mais baixos no grupo da memantina quando comparado ao placebo. $O$ estudo dos resultados dos custos sociais realizados pelo TPP foi confirmado pelas análises feitas pelo LOCF e pela ITT, evidenciando uma média de diferença mensal de \$US 840,40 em favor da memantina, sendo a diferença estatisticamente significante $(p=0,01)$.

\section{Discussão}

Os achados sugerem que os pacientes com DA de estágios moderado a grave podem experimentar benefícios com o tratamento antiglutaminérgico, diminuindo a progressão da deterioração característica da doença, principalmente no que diz respeito à atividade da vida diária. A utilização da memantina, na dose de $20 \mathrm{mg}$ por dia, pode reduzir tempo e custo dos gastos por parte dos cuidadores e, ao mesmo tempo, melhorar os domínios global, funcional e comportamental dos pacientes portadores de DA. A eficácia, a segurança e a tolerabilidade do uso da memantina em pacientes portadores de DA nos estágios de moderado a grave foram demonstradas na avaliação dos quatro estudos selecionados. Todos os artigos analisados evidenciaram benefícios do uso da memantina nas doses de $10 \mathrm{mg}$ ou $20 \mathrm{mg}$ ao dia, quando comparada com o placebo, apresentando melhora da capacidade funcional, das atividades da vida diária e dos distúrbios comportamentais, com diferenças estatisticamente significativas. A melhora da cognição, entretanto, não foi superior à do placebo em dois estudos (Reisberg et al., 2003; Wimo et al., 2003).

A memantina aumenta a autonomia do paciente portador de DA, principalmente na habilidade de levantarse, mover-se de forma independente, tomar banho ou ir ao banheiro, vestir-se, orientar-se no espaço, realizar atividades em grupos e interessar-se por hobbies (Winblad e Poritis, 1999). Portanto as pressões e o estresse sofridos pelo cuidador diminuirão, bem como o tempo gasto com o paciente e o risco de depressão, aumentando então a sua qualidade de vida. A análise dos estudos não demonstrou relevância quanto aos efeitos colaterais; ao contrário, os efeitos adversos foram até maiores no grupo placebo do que no da memantina, sugerindo que os prováveis efeitos colaterais não eram decorrentes da droga, mas sim da própria gravidade da doença.

A relação custo/benefício favorece o uso da memantina, pois além de reduzir os custos totais com o paciente portador de DA, melhora a capacidade funcional e os distúrbios do comportamento (Wimo et al., 2003). Assim, o gasto obtido para a manutenção medicamentosa é compensado por redução dos custos adicionais, melhora do paciente e aumento da qualidade de vida do paciente e do cuidador.

Todos os estudos avaliaram memantina versus placebo num período de 28 semanas. Entretanto, Reisberg et al. avaliaram a eficácia da memantina em longo prazo, realizando uma extensão de 24 meses do estudo duplo-cego, randomizado e placebo-controlado (Reisberg et al., 2006). Os pacientes, que antes recebiam placebo, passaram a receber memantina $20 \mathrm{mg} /$ dia e apresentaram um benefício estatisticamente significante, revelando menor grau de declínios funcional, global e cognitivo, quando em comparação com o placebo durante estudo duplo-cego $(p<0,05)$. Na fase de extensão os efeitos adversos dos pacientes tratados com memantina tiveram perfil similar ao dos pacientes do estudo duplo-cego. A extensão da terapia com memantina por mais 24 semanas também se mostrou benéfica para pacientes portadores de DA nos estágios moderado a severo.

Uma metanálise publicada no Cochrane Database of Systematic Reviews (McShane et al., 2006) incluindo estudos não-publicados sugere que a memantina mostrou-se benéfica para pacientes com DA nos estágios moderado a grave, quando avaliada por SIB, ADCS-ADLsev, NPI e CIBIC-Plus. Houve progresso na melhora clínica, cognitiva e funcional, com resultados estatisticamente significantes em relação ao placebo. Pacientes 
que receberam memantina desenvolveram discretamente menos agitação, quando em comparação com placebo $(p<0,04)$ na doença leve. A agitação foi um pouco maior nos pacientes com doença moderada a grave, porém permaneceu menor do que nos que receberam placebo $(p<0,005)$. Parece, portanto, que esse efeito é conseqüência da própria doença.

\section{Referências}

Arnold K, Lehfeld H, Mosler TH, Erzigkeit H. (1998) Scale properties of the D-scale in severe dementia. $6^{\text {th }}$ Int Cong Alzheimer's Dis. 18-23 July, Amsterdam.

Danysz W, Parsons CG, Möbius H-J, et al. Neuroprotective and symptomatological action of memantine relevant for Alzheimer's disease a unified hypothesis on the mechanism of action. Neurotoxicity Res, 2: 85-98, 2000.

Fonnum F. Glutamate: a neurotransmitter in mammalian brain. J. Neurochem, 42(1): 1-11, 1984.

Henderson AS, Jorm AF. Definition and epidemiology of dementia: a review. In: Maj M, Sartorius N, editors. Dementia. 2 ed. Chichester, England: John Wiley; 2002, p. 1-33.

Khachaturian ZS. Plundered memories. Sciences, 37(4): 20-5, 1997.

McKhann G, Drachman D, Folstein M, et al. Clinical diagnosis of Alzheimer's disease: report of the NINCDS-ADRDA Work Group under the auspices of Department of Health and Human Services Task Force on Alzheimer's Disease. Neurology. 34: 939-44, 1984.

McShane R, Sastre AA, Minakaran N. Memantine for dementia. 2 ed. The Cochrane Database of Systematic Reviews; 2006.

Orrego F, Villanueva S. The chemical nature of the main central excitatory transmitter: a critical appraisal based upon release studies and synaptic vesicle localization. Neuroscience, 56: 539-55, 1993.
Sabe-se que a doença de Alzheimer é progressiva e que as medicações antidemenciais têm uma função apenas paliativa. Até o presente momento a memantina foi testada e se mostrou superior ao placebo por um período de 28 semanas, mas seu uso prolongado carece de evidências científicas em estudos controlados.
Reisberg B, Doody R, Stoffler A, et al. Memantine in moderate-to-severe Alzheimer's disease. New Engl J Med, 348: 1333-41, 2003.

Reisberg B, Doody R, Stoffler A, Schmitt F, Ferris S, Mobius HJ. A 24-week open-label extension study of memantine in moderate to severe Alzheimer's disease. Archives of Neurology, 63(1): 46-54, 2006.

Rive $B$, et al. Memantine enhances autonomy in moderate to severe Alzheimer's disease. Int J Geriatr Psychiatry, 19: 458-64, 2004.

Van der Kam P, Mol F, Wimmers MFHC. Beoordelingsschaal voor oudere patienten (BOP). Van Loghum Slaterus, The Netherlands; 1971.

Wimo A, Wetterholm AL, Mastey V, Winblad B. Evaluation of the healthcare resource utilization and caregiver time in anti-dementia drug trials a quantitative battery. In: Wimo A, Jonsson B, Karlsson $G$, Winblad B, editors. Health economics of dementia. Chichester, UK: John Wiley; 1998, p. 465-99.

Wimo A, Winblad B, Stoffler A, et al. Resource utilization and cost analysis of memantine in patients with moderate to severe Alzheimer's disease. Pharmacoeconomics, 21(5): 1-14, 2003.

Winblad B, Poritis N. Memantine in severe dementia: results of the MBEST study (Benefit and Efficacy in Severely demented patients during Treatment with memantine). Int J Geriat Psychiatry, 14: 135-46,1999. 